\title{
A Phase 2 Randomized Controlled Trial of the Efficacy and Safety of Cannabidivarin as Add-on Therapy in Participants with Inadequately Controlled Focal Seizures
}

\author{
Martin J. Brodie, ${ }^{1, *}$ Piotr Czapinski, ${ }^{2}$ Ladislav Pazdera, ${ }^{3}$ Josemir W. Sander, ${ }^{4-6}$ Manuel Toledo, ${ }^{7}$ Mariana Napoles, \\ Farhad Sahebkar, ${ }^{9}$ and Ashley Schreiber ${ }^{8}$; on Behalf of the GWEP1330 Study Group ${ }^{\dagger}$
}

\begin{abstract}
Objective: We assessed the efficacy, safety, and tolerability of cannabidivarin (CBDV) as add-on therapy in adults with inadequately controlled focal seizures.

Materials and Methods: One hundred and sixty-two participants (CBDV $n=81$; placebo $n=81$ ) were enrolled. After a 4-week baseline, participants titrated from 400 to $800 \mathrm{mg}$ CBDV twice daily (b.i.d.) (or placebo) over 2 weeks, followed by 6 weeks stable dosing (at $800 \mathrm{mg}$ b.i.d.) and a 12-day taper period. The primary endpoint was the change from baseline in focal seizure frequency during the 8-week treatment period. Secondary endpoints included additional efficacy measures relating to seizures, physician- and participant-reported outcomes, change in the use of rescue medication, cognitive assessments, and safety.

Results: Median baseline focal seizure frequencies were 17-18 per 28 days in both groups, and similar reductions in frequency were observed in the CBDV (40.5\%) and placebo (37.7\%) groups during the treatment period (treatment ratio [\% reduction] CBDV/placebo: 0.95 [4.6]; confidence interval: 0.78-1.17 [ -16.7 to 21.9]; $p=0.648$ ). There were no differences between the CBDV and placebo groups for any seizure subtype. There were no significant treatment differences between CBDV and placebo groups for any of the secondary efficacy outcome measures. Overall, 59 (72.8\%) of participants in the CBDV group and 39 (48.1\%) in the placebo group had $\geq 1$ treatment-emergent adverse event (AE); the 3 most common were diarrhea, nausea, and somnolence. The incidence of serious AEs was low (3.7\% in the CBDV group vs. $1.2 \%$ in the placebo group). There was little or no effect of CBDV on vital signs, physical examination, or electrocardiogram findings. Elevations in serum transaminases (alanine aminotransferase or aspartate aminotransferase) to levels $>3 \times$ upper limit of normal occurred in three participants taking CBDV (two discontinued as a result) and one taking placebo; however, none met the criteria for potential Hy's Law cases.

Conclusion: It is likely the $40.5 \%$ seizure reduction with CBDV represents an appropriate pharmacological response in this population with focal seizures. The placebo response was, however, high, which may reflect the participants' expectations of CBDV, and a treatment difference from placebo was not observed. CBDV was generally well tolerated. Clinical Trial Registration number: NCT02365610.
\end{abstract}

Keywords: antiepileptic drug; cannabinoid; GWP42006; epilepsy

\footnotetext{
'Epilepsy Unit, University of Glasgow, Glasgow, United Kingdom.

${ }^{2}$ Epilepsy and Migraine Treatment Center, Krakow, Poland.

${ }^{3}$ Vestra Clinics - Dedicated Research Clinics, Rychnov nad Kneznou, Czech Republic.

${ }^{4}$ NIHR University College London Hospitals Biomedical Research Centre, UCL Queen Square Institute of Neurology, London, WC1N 3BG, United Kingdom.

${ }^{5}$ Chalfont Centre for Epilepsy, Chalfont St. Peter, United Kingdom.

${ }^{6}$ Stichting Epilepsie Instellingen Nederland (SEIN), Heemstede, Netherlands.

${ }^{7}$ Epilepsy Unit, Neurology Department, Vall d'Hebron Hospital, Barcelona, Spain.

${ }^{8} \mathrm{GW}$ Research Ltd, Cambridge, United Kingdom.

${ }^{9}$ Greenwich Biosciences, Inc., Carlsbad, California, USA.

${ }^{\dagger}$ For members, see Acknowledgments section.

*Address correspondence to: Martin J. Brodie, MD, Epilepsy Unit, University of Glasgow, Glasgow G12 8QQ, United Kingdom, E-mail: martin.brodie@glasgow.ac.uk
}

(c) Martin J. Brodie et al. 2021; Published by Mary Ann Liebert, Inc. This Open Access article is distributed under the terms of the Creative Commons Attribution Noncommercial License [CC-BY-NC] (http://creativecommons.org/licenses/by-nc/4.0/) which permits any noncommercial use, distribution, and reproduction in any medium, provided the original author(s) and the source are cited. 


\section{Introduction}

Cannabidivarin (CBDV) is a phytocannabinoid derived from the Cannabis sativa L. plant, ${ }^{1}$ which is currently being developed for the treatment of several conditions, including epilepsy. CBDV lacks appreciable affinity and activity at the human cannabinoid receptor, $\mathrm{CB}_{1}$, responsible for the euphoric effects associated with cannabis use. ${ }^{2}$ CBDV has been shown to reduce epileptiform activity in vitro, ${ }^{3}$ and to exert anticonvulsant effects in a broad range of seizure models in vivo, without significantly affecting normal motor function. ${ }^{2,4}$ CBDV was also found to ameliorate the induction of neuronal degeneration in seizure models in immature rats, suggesting potential therapeutic value. ${ }^{4} \mathrm{~A}$ role has been suggested for the transient receptor potential vanilloid receptor 1 in CBDVs anticonvulsant effects. ${ }^{4}$

To date, there have been limited clinical data on CBDV. Before this trial, the effect of concomitant antiseizure medications (ASMs) on the safety and pharmacokinetics of CBDV (after 2 weeks dosing at $400 \mathrm{mg}$ twice daily [b.i.d.]) was assessed. This was done to assess potential for drug interactions with available ASMs. No potential interactions were found, and CBDV had an acceptable safety profile (NCT02369471, data on file).

The current trial aimed to evaluate the efficacy and safety of CBDV administered concomitantly with other ASMs, compared with placebo, to treat focal seizures.

\section{Materials and Methods}

Participants

Eligible participants were adults aged between 18 and 65 years (inclusive) with a documented history of focal epilepsy (documented by compatible electroencephalogram and clinical history). Eligible participants had seizures despite prior treatment with at least two ASMs and were receiving one to three concomitant ASMs. They were required to have experienced $\geq 1$ weekly seizure during the 28 days preceding screening and 1 weekly seizure during the 28-day baseline. Countable seizures included the following: focal motor seizures without impairment of consciousness or awareness, focal seizures with impairment of consciousness or awareness, and focal seizures evolving to bilateral convulsive seizures. All medications or interventions for epilepsy had to be stable for 4 weeks before screening and throughout the trial. More detailed inclusion criteria can be found in Supplementary Data.

\section{Trial design}

This phase 2 randomized, double-blind, placebocontrolled trial was conducted between March 2016 and September 2017. It included 34 trial sites (5 in the Czech Republic, 7 in Hungary, 1 in Italy [no participants screened], 12 in Poland, 4 in Spain, and 5 in the United Kingdom).

Participants were randomized 1:1 to receive CBDV or placebo. There was a 4 -week baseline period, a 2week dose escalation period in which participants received $400 \mathrm{mg}$ CBDV b.i.d. for 7 days (or placebo equivalent), then $600 \mathrm{mg}$ CBDV b.i.d. for 7 days (or placebo equivalent). This was followed by a 6 -week stable treatment period at the target dose of $800 \mathrm{mg}$ b.i.d. (or placebo equivalent) and a 12-day taper period. Individual dose escalation was subject to the investigator assessment of safety and tolerability. Participants were required to attend eight trial visits (screening [days -28 to 3], randomization [day 1], during treatment [days $8 \pm 3,15 \pm 3$, $29 \pm 3$, and $43 \pm 3$ ], end of treatment [day $57 \pm 3$ ], and

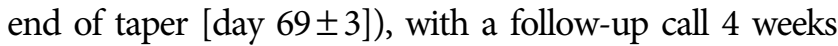
( \pm 3 days) after the last dose.

$\mathrm{CBDV}$ and placebo were oral solutions containing $50 \mathrm{mg} / \mathrm{mL}$ CBDV and excipients or excipients only (placebo). Participants were instructed to fast for at least $2 \mathrm{~h}$ before taking the trial compounds and for 30 min afterward.

\section{Randomization and blinding}

Randomization occurred before treatment administration on day 1 . Allocation to each treatment group was conducted through an interactive voice/web response system, according to a randomization schedule produced by an independent statistician. The randomization schedule was held centrally and not divulged to any other person involved in the trial until the database was locked and unblinding authorized by the relevant sponsor personnel. Investigators, participants, and the sponsor were blinded to the treatment allocation.

\section{Outcome measures}

The primary objective was to evaluate the efficacy of CBDV by measuring the change from 4 -week baseline in focal seizure frequency during the 8-week treatment period for those taking CBDV compared with placebo. Secondary objectives were to evaluate the effects of CBDV compared with placebo on other measures of efficacy, including summary of treatment responders, proportion of participants with reductions in focal seizure frequency [ $>0 \%$ and $\leq 25 \%,>25 \%$ and $\leq 50 \%$, $>50 \%$ and $\leq 75 \%$, and $>75 \%$ and $\leq 100 \%$, change in seizure subtype frequency, change in composite 
seizure score (the weighted sum of seizure subtypes, where weights were the severity of seizure subtypesType 1 seizures [least severe] were graded as "1," Type 2 seizures as " 2 ," and Type 3 seizures [most severe] as " 3 "; see trial procedures for seizure type definition), change in number of focal seizure free days, change in rescue medication use, Physician Global Impression of Change (PGIC), and Subject Global Impression of Change (SGIC). Safety was monitored through adverse event (AE) recording, clinical laboratory tests, vital signs, physical examination, urinalysis, and 12-lead electrocardiograms (ECGs). Suicidal behavior and ideation were monitored using the Columbia Suicide Severity Rating Scale, ${ }^{5}$ and withdrawal symptoms were monitored using the cannabis withdrawal scale at randomization (day 1), the end of treatment, 1 day after the last dose, and 7, 14, 21 , and 28 days after the last dose of CBDV. ${ }^{6}$ Finally, to investigate cognitive function at the start and end of treatment, the Trail Making Test (Halstead-Reitan), Lafayette Grooved Pegboard, and Symbol Digit Modalities Test were employed.

\section{Trial procedures}

Safety was monitored throughout the trial, using the parameters above.

Participants recorded seizure data in a diary throughout the trial. Seizures were classified into four categories: Type 1: focal seizures, which did not impair consciousness or awareness and had a motor component; Type 2: focal seizures, which impaired consciousness or awareness; Type 3: focal seizures, which evolved to bilateral convulsive seizures; and Type 4: other (seizures other than those listed above, i.e., focal seizures without impairment of consciousness or awareness and without an observable motor component). Seizure data were used for analysis of the primary (Types 1-3) and several secondary endpoints. An independent committee of experts from the Epilepsy Study Consortium reviewed the individuals' documented seizure history and subtype classification.

At the end of treatment (day 57), the PGIC and SGIC were administered. Both are 7-point Likert scales with markers from "very much improved" to "very much worse." Physicians were asked to assess changes in the participants' general functional abilities since starting treatment, and participants were asked to rate their overall condition since starting treatment. Before starting treatment (day 1), participants wrote a brief description of their overall condition as a memory aid for completion of the SGIC at treatment end.
A cognitive assessment battery to assess coordination, attention, and visual-spatial integration was performed at the start and end of treatment (days 1 and 57), including Trail Making Test (Halstead-Reitan), Lafayette Grooved Pegboard, and Symbol Digit Modalities Test.

\section{Statistical analysis}

The trial intended to enroll 140 adults randomized in a 1:1 ratio to receive CBDV or placebo (70 per group). It was assumed that those in the placebo group would experience a mean reduction in focal seizure frequency of $15 \%$ (from baseline), meaning a sample size of 70 per group would allow detection of a difference of $35 \%$ between treatments. This was based on a standard deviation (SD) of $63 \%$, using a 2 -sided $5 \%$ significance level and $90 \%$ power.

There were two analysis sets for the reported trial data: intention to treat (ITT) and safety. Both included all participants who received at least one dose of CBDV or placebo. Efficacy endpoints were described and compared by group. All statistical analysis was 2 -tailed and carried out at the $5 \%$ level of significance. The primary outcome variable for the determination of efficacy was the change from baseline in focal seizure frequency per 28 days during the 8-week treatment period. Data were analyzed using percentage change from baseline, if found to be normally distributed; however, due to non-normality of the seizure data, negative binomial regression analyses were conducted, where treatment ratios $<1$ indicate a difference in favor of CBDV. Safety data were summarized using appropriate summary statistics.

\section{Compliance with ethical standards}

This trial was conducted in accordance with International Council for Harmonisation Good Clinical Practice guidelines and ethical principles that have their origin in the Declaration of Helsinki. The protocol was approved by all relevant local Ethics Committee, and all participants provided written informed consent. The trial is registered on the clinicaltrials.gov website.

\section{Results}

\section{Disposition of participants}

A total of 162 participants were randomized to receive CBDV $(n=81)$ or placebo $(n=81)$, and were included in the ITT and safety analysis sets. Participant 


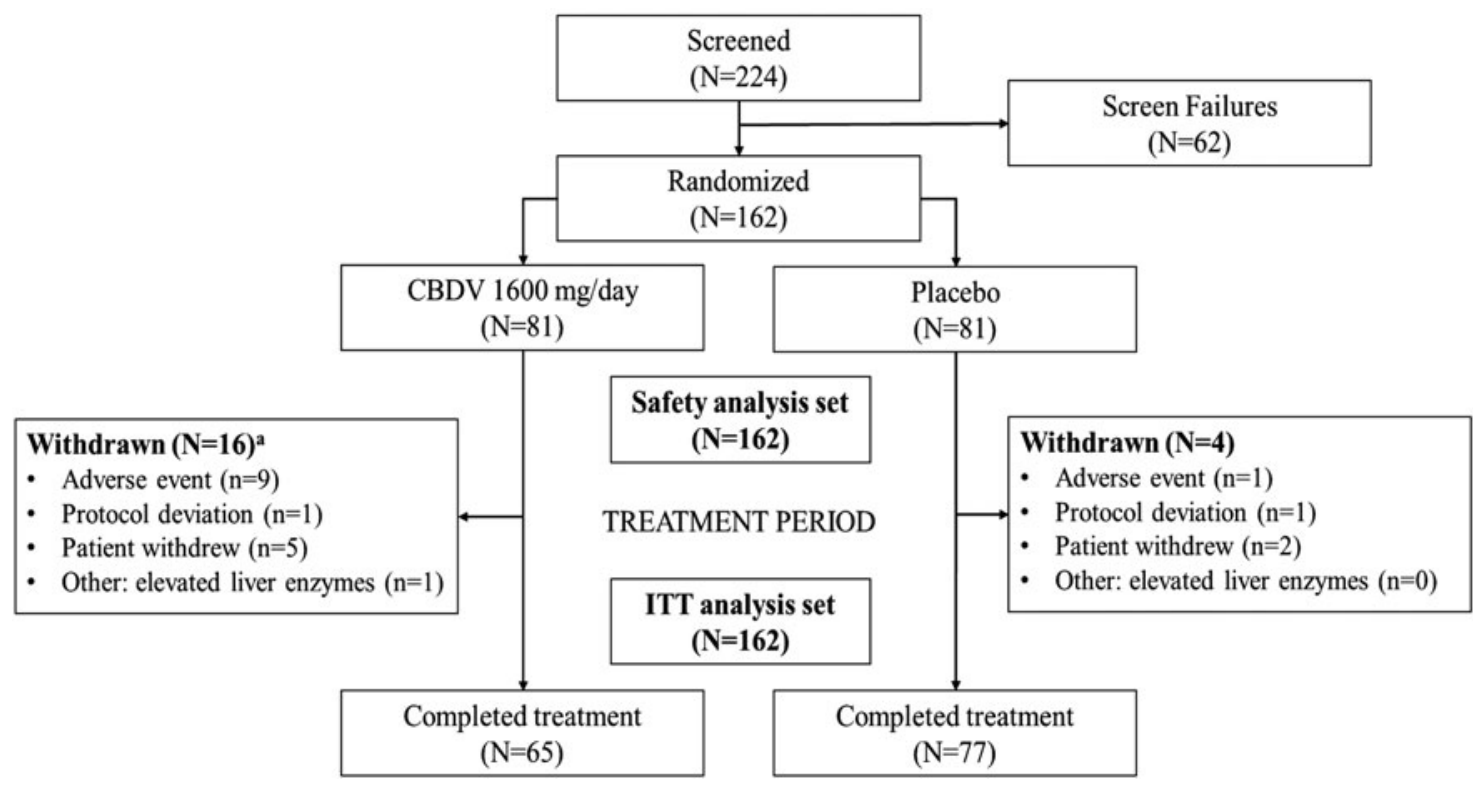

FIG. 1. Disposition of participants. ${ }^{a}$ One participant in the CBDV group discontinued treatment on day 32 due to an adverse event of severe diarrhea and did not restart treatment after adverse event resolution; however, they attended the end-of-treatment visit and was reported as completing the trial. CBDV, cannabidivarin; ITT, intention to treat.

disposition is summarized in Figure 1. In the CBDV group, $16(20 \%)$ participants withdrew early from the trial; $9(11 \%)$ due to AEs, 5 (6\%) withdrew consent, 1 (1\%) due to a protocol deviation, and 1 (1\%) experienced elevated liver enzymes so was withdrawn by the investigator. In the placebo group, $4(5 \%)$ withdrew early from the trial; $1(1 \%)$ due to an AE, 2 (2\%) withdrew consent, and 1 (1\%) due to a protocol deviation.

Demographics are summarized in Table 1 and were similar between the two groups. The median number of prior ASMs was similar between treatment groups ( 4 for CBDV and 5 for placebo), and the median number of concomitant ASMs taken during the trial was 3 in both treatment groups. The most common concomitant ASMs taken were levetiracetam (43\% CBDV vs. $48 \%$ placebo), lamotrigine ( $42 \%$ vs. $43 \%$ ), valproic acid ( $40 \%$ vs. $33 \%)$, lacosamide ( $28 \%$ vs. $32 \%)$, and carbamazepine ( $25 \%$ vs. $31 \%)$. Six (7\%) participants randomized to $\mathrm{CBDV}$ and five $(6 \%)$ randomized to placebo had an active vagus nerve stimulator in situ throughout the trial.

The median number of seizures during baseline per 28 days was 18.1 in the CBDV group versus 17.0 in the placebo group. Overall, the mean time since onset of
Table 1. Demographics and Characteristics (Safety Analysis Set)

\begin{tabular}{|c|c|c|}
\hline & CBDV $(N=81)$ & Placebo $(N=81)$ \\
\hline \multicolumn{3}{|l|}{ Sex } \\
\hline Male, $N(\%)$ & $34(42)$ & $38(47)$ \\
\hline Female, $N(\%)$ & $47(58)$ & $43(53)$ \\
\hline \multicolumn{3}{|l|}{ Race } \\
\hline White/Caucasian, $N(\%)$ & $80(99)$ & $81(100)$ \\
\hline Asian, $N(\%)$ & $1(1)$ & 0 \\
\hline Age (years), mean (SD) & $36.0(11)$ & $36.1(13)$ \\
\hline Weight $(\mathrm{kg})$, mean (SD) & $69.7(17)$ & $73.8(19)$ \\
\hline \multicolumn{3}{|l|}{ Country } \\
\hline Czech Republic, $N(\%)$ & $10(12)$ & $13(16)$ \\
\hline Hungary, $N(\%)$ & $13(16)$ & $11(14)$ \\
\hline Poland, $N(\%)$ & $50(62)$ & $48(59)$ \\
\hline Spain, $N(\%)$ & $4(5)$ & $4(5)$ \\
\hline United Kingdom, $N(\%)$ & $4(5)$ & $5(6)$ \\
\hline $\begin{array}{l}\text { Baseline focal seizure } \\
\text { frequency, mean (SD) }\end{array}$ & $38.7(54)$ & $41.7(74)$ \\
\hline \multicolumn{3}{|l|}{ Baseline seizure frequency } \\
\hline Type 1 , mean $(S D)^{a}$ & $33.5(64)[N=34]$ & $25.5(35)[N=42]$ \\
\hline Type 2 , mean $(S D)^{a}$ & $23.8(30)[N=66]$ & $30.9(72)[N=62]$ \\
\hline Type 3, mean (SD) ${ }^{a}$ & $11.0(12)[N=38]$ & $12.9(25)[N=30]$ \\
\hline Type 4, mean (SD) ${ }^{a}$ & $16.5(36)[N=9]$ & $8.1(19)[N=10]$ \\
\hline
\end{tabular}

aType 1: focal seizures, which did not impair consciousness or awareness and had a motor component; Type 2: focal seizures, which impaired consciousness or awareness; Type 3: focal seizures, which evolved to bilateral convulsive seizures; and Type 4: other (seizures other than those listed above, that is, focal seizures without impairment of consciousness or awareness and without an observable motor component).

CBDV, cannabidivarin; $N$, number of participants; $S D$, standard deviation. 
seizures was 21.5 years (SD: 10.8 ) in the CBDV group and 20.9 years (SD: 12.1 years) in the placebo group. The number of participants with each type of focal seizure at baseline (Types 1-4) is presented in Table 1, alongside total seizure frequency by type at baseline. Thirteen participants had previously experienced status epilepticus; the mean time since last episode was 12.5 years; 17.1 years (range: $1.1-41.5$ years) in the CBDV group; and 9.6 years (range: 1.1-26.5 years) in the placebo group.

\section{Drug exposure}

The mean number of dosing days reported during the treatment period was 49.3 days in the CBDV group and 54.5 days in the placebo group. In both treatment groups, the median number of dosing days during the treatment period was 57.0 days. The mean modal daily CBDV dose during the treatment period was $1480 \mathrm{mg} /$ day.

\section{Safety}

A summary of treatment-emergent adverse events (TEAEs) is presented in Table 2. Most AEs were mild (26\% CBDV; $30 \%$ placebo) or moderate (40\% CBDV; $12 \%$ placebo) in intensity. The most common AE leading to treatment discontinuation was diarrhea, reported by $4(5 \%)$ participants taking CBDV (vs. none taking placebo); all recovered. Three (4\%) participants taking CBDV discontinued treatment due to a maculopapular rash; all recovered. All other AEs leading to treatment discontinuation were reported by $\leq 1$ participant per group.

Table 2. Summary of Treatment-Emergent Adverse Events

\begin{tabular}{lcc}
\hline & CBDV $\mathbf{N = 8 1}$ & Placebo $\mathbf{N = 8 1}$ \\
\cline { 2 - 3 } & & No. of participants (\%) \\
\hline TEAE & & \\
All TEAEs & $59(73)$ & $39(48)$ \\
TEAEs leading to treatment cessation & $11(14)$ & $2(3)$ \\
Serious TEAEs & $3(4)$ & $1(1)$ \\
Mild TEAEs & $21(26)$ & $24(30)$ \\
Moderate TEAEs & $32(40)$ & $10(12)$ \\
Severe TEAEs & $6(7)$ & $5(6)$ \\
TEAEs reported by $>5 \%$ participants in either treatment group \\
Diarrhea & $20(25)$ & $6(7)$ \\
Nausea & $8(10)$ & $8(10)$ \\
Abdominal pain upper & $5(6)$ & $1(1)$ \\
Somnolence & $12(15)$ & $2(3)$ \\
Headache & $7(9)$ & $6(7)$ \\
Dizziness & $5(6)$ & $3(4)$ \\
Decreased appetite & $2(3)$ & $6(7)$ \\
\hline
\end{tabular}

TEAE, treatment-emergent adverse event.
AEs leading to treatment discontinuation were serious for 2 (2\%) participants taking CBDV. One participant taking CBDV experienced a serious TEAE of intoxication, pharmacologic, symptoms of which included severe ataxia, nystagmus, diplopia, dysarthria, mild confusion, elevated liver transaminases, and arterial hypotension. The TEAE began on day 31 (midway through maintenance dosing), led to treatment discontinuation after 5 days, and resolved 10 days after treatment discontinuation. The other participant experienced serious AEs of diarrhea, azotemia, and acute renal failure concurrently on day 33 (midway through maintenance dosing), which resolved after treatment discontinuation due to diarrhea. One other participant in the CBDV group experienced a serious AE but did not discontinue. The participant had a history of cholelithiasis and experienced an acute episode during a scheduled cholecystostomy during the taper period.

In the placebo group, one participant experienced status epilepticus 5 days into the taper period, which resolved after 1 day.

No participants in either treatment group reported suicidal behavior on the columbia-suicide severity rating scale during or at the end of treatment. In terms of the cannabis withdrawal scale (CWS), a reduction in score indicates an improvement in withdrawal symptoms. Post-treatment changes in overall CWS scores decreased from baseline in both treatment groups; therefore, there were no signs of withdrawal in either treatment group.

There was little or no effect of CBDV on vital signs, physical examination findings, or ECGs. Overall changes in vital signs or ECG that met predefined criteria for clinical significance were generally similar between the treatment groups. Elevations in serum transaminases (alanine aminotransferase or aspartate aminotransferase) to levels $>3 \times$ upper limit of normal occurred in three participants taking CBDV (two participants discontinued as a result) and one taking placebo; however, none met the criteria for potential Hy's Law cases. All participants with elevated serum transaminases were taking at least one concomitant medication associated with transaminase elevations, such as carbamazepine, lacosamide, lamotrigine, clarithromycin, or valproic acid. In the CBDV treatment group, no participants shifted from having normal aspartate aminotransferase levels at baseline to high levels ( > upper limit of normal) at the end of treatment compared with $2(2 \%)$ participants in the placebo group. There were no significant changes, either 
between baseline and the end of treatment, or between treatment groups in any of the cognitive assessments: Trail Making Test (Halstead-Reitan), Lafayette Grooved Pegboard, or the Symbol Digit Modalities Test.

\section{Efficacy}

For the primary endpoint, similar reductions in focal seizure frequency were observed in the CBDV (41\%) and placebo (38\%) groups during the treatment period. The treatment difference was in favor of CBDV but was not statistically significant (Table 3 ). A summary of effect modifier analyses of the primary efficacy endpoint by subgroup is shown in Figure 2 . The treatment ratio was $\sim 1$ for all subgroups, and in all cases the $95 \%$ confidence interval (CI) values crossed 1 ; all interaction $p$-values were $>0.1$, suggesting no strong interaction between any of the subgroups analyzed.

Reductions in specific seizure types were inconsistent, and no difference between treatments reached statistical significance. Accordingly, no statistical difference was found in the seizure composite score. The proportion of participants with a $\geq 25 \%, \geq 50 \%, \geq 75 \%$, or $100 \%$ reduction in seizure frequency was similar between treatment groups.

Participants in the CBDV group achieved a mean gain of 5 days of total seizure freedom over the treatment period versus 4 days in the placebo group; this difference was not statistically significant (treatment difference:

$\begin{aligned} & \text { Table 3. Negative Binomial Regression Analysis of Focal } \\ & \text { Seizure Counts During Baseline and Treatment Periods } \\ & \text { (Intention-to-Treat Analysis Set) }\end{aligned}$
$\begin{gathered}\text { CBDV } \\ N=81\end{gathered}$

${ }^{\mathrm{a}}$ Improvement from baseline.

${ }^{\mathrm{b}}$ Result in favor of CBDV.

$\mathrm{Cl}$, confidence interval.
0.9; $95 \%$ CI: -0.5 to $2.3 ; p=0.195)$. Rescue medication use was relatively infrequent and remained unchanged.

Physicians reported $56 \%$ of participants randomized to CBDV and placebo as having improved from baseline (very much, much, or slightly improved) in their general functional abilities at the end of treatment. The respective proportions of participants who reported any improvement in overall condition from baseline were $61 \%$ in the CBDV group and $64 \%$ in the placebo group. Similar proportions in each treatment group fell into each of the seven change categories for the PGIC and SGIC. There was no statistical difference between the treatment groups for either the PGIC (odds ratio $[\mathrm{OR}]=0.96$; 95\% CI: $0.55-1.69 ; p=0.895$ ) or SGIC (OR $=1.05$; 95\% CI: $0.59-1.86 ; p=0.866)$.

\section{Discussion}

This trial showed that add-on CBDV treatment in participants with focal seizures had an acceptable safety profile, but there was no difference in seizure reductions between the CBDV and placebo treatment groups after 8 weeks of treatment.

An adult population was targeted given the limited human exposure data available for CBDV. Unlike cannabidiol, plants rich in CBDV are rare in nature. A single Phase 1 study in adult healthy volunteers had been carried out before the start of this study. In addition, at the time this study was run, animal juvenile toxicology data were not available. The dose was selected based on the dose range tested in the Phase 1 study, in which a maximum of $800 \mathrm{mg}$ once daily for 5 days was used. In this study, doses of $800 \mathrm{mg}$ b.i.d. were administered to maximize exposure. The dose selected was supported by the animal toxicology data.

We observed a large placebo effect. The magnitude of the placebo effect in human epilepsy trials has been previously documented in meta-analyses, where estimates of the placebo response were reported to be $13 \%$ per data pooled from 1887 placebo-treated individuals ${ }^{7}$ and ranged from $9 \%$ to $17 \%$ for $50 \%$ responders in a further review of randomized controlled trials. ${ }^{8}$ In the current trial, $36 \%$ of participants taking CBDV were $50 \%$ responders versus $33 \%$ in those taking placebo. This is a larger placebo effect than has been previously reported. ${ }^{7,8} \mathrm{~A}$ large heterogeneity in placebo response rates has also been noted between trials, ${ }^{9}$ and a correlation within trials between the placebo and drug response has been reported. ${ }^{10}$ These findings suggest that the magnitude of the placebo effect may be influenced by the trial design, location, and population. 


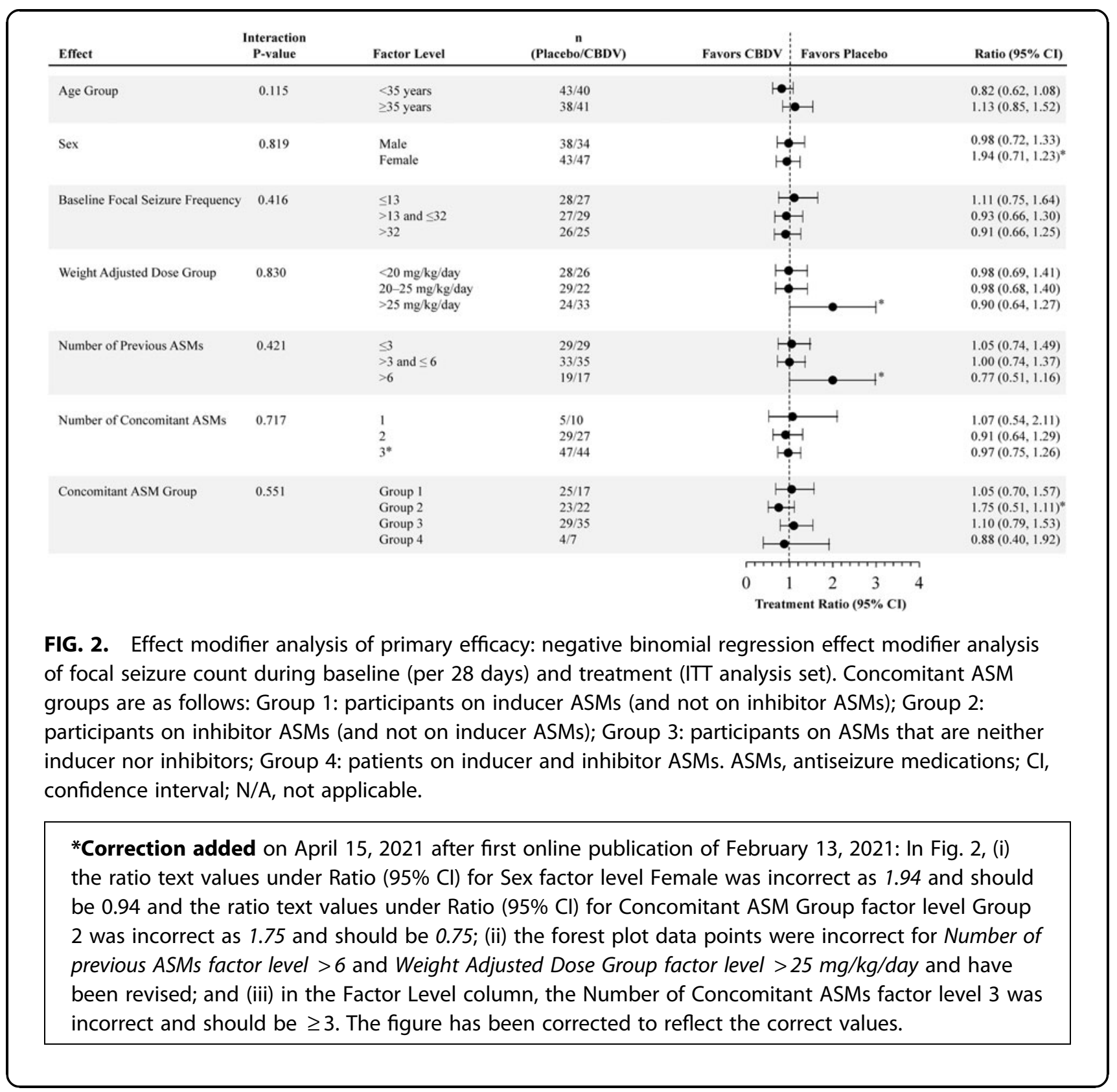

Causes of high placebo response rates in epilepsy trials have been proposed as classical conditioning, the Hawthorne effect, high expectations of the trial drug, regression to the mean, and natural fluctuations of the disease. ${ }^{10,11}$ In the current trial, baseline seizure frequency was comparable between groups, and effect modifier analyses indicated that there was little interaction between groups in relation to baseline seizure frequency. This suggests that regression to the mean is unlikely to be the main contributor for the observed effects.
In terms of trial design, a factor that may have contributed to the overall response is the relatively short evaluation period. The baseline data consisted of 4 weeks, and the treatment period was 8 weeks. This was a proof-of-concept trial, and the aim was to determine efficacy while minimizing the period of exposure. In a retrospective analysis of pregabalin data, it has been suggested that a 4-week baseline and 3-week treatment could be enough to determine efficacy for a highly efficacious drug. ${ }^{12}$ Given the unknown efficacy 
of CBDV and the slow dose escalation adopted, CBDV was generally well tolerated, with low incidence rates of serious TEAEs.

This study has some important limitations. Many factors can potentially affect the results of clinical studies in people with epilepsy, including heterogeneity of the data across geographic regions or study centers, and baseline characteristics of the participants. Unfortunately, the number of participants included in this trial was too small to conclusively evaluate these factors.

\section{Conclusions}

CBDV was generally well tolerated, but efficacy of CBDV in the treatment of focal seizures was not demonstrated. A high placebo response was observed. Further studies of ASMs should consider trials designed to control for the large placebo effect often observed in this population. The neuromodulatory potential of CBDV is still under investigation.

\section{Acknowledgments}

We are indebted to the individuals who took part in the trial, as well as to the staff at the clinical research sites. We thank Dr. Lesley Taylor of Alchemy Medical Writing Ltd for medical writing and editorial support, funded by Greenwich Biosciences, Inc. The study group investigators made significant contributions to the planning and/or execution of the study. Study group members (including authors) confirming approval to list their name are provided below in alphabetical order:

Czech Republic: Tomas Nezadal (INEP medical s.r.o., Křižíkova 264/22, 18600 Praha 8); Jana Slonkova (JS-GYNAM s.r.o., Studentská 1155/14, 73601 Havírov). Hungary: Anna Altman (Servus Salvus K ft., Szilagyi Erzsebet fasor 17-21. fel.3., Budapest); Kálmán Füle (Bács-Kiskun Megyei Kórház, SZTE ÁOK Oktató Kórháza, H-6000 Kecskemét, Nyíri út 38); Ágnes Horváth (Markusovszky Egyetemi, EEG Diagnosztikai es Epilepszia Centrum, Markusovszkky L. u. 5. Szombathely); Anna Kelemen (Országos Klinikai Idegtudományi Intézet, H-1145 Budapest, Amerikai út 57); Sámuel Komoly (Pécsi Tudományegyetem, Klinikai Központ Neurológiai Klinika, Ret u. 2., Pecs). Poland: Marta Banach (Specjalistyczny Gabinet Neurologiczny Marta Banach, Plac Lasoty 4, 30-534 Kraków); Iwona Kurkowska-Jastrzebska (Institute of Psychiatry and Neurology, Second Department of Neu- rology, ul. Sobieskiego 9, Warszawa); Paweł Lisewski (Vitamed Gałaj I Cichomski Spółka Jawna, Ul. Kościuszki 35, Bydgoszcz; Jolanta Zagórska (Centrum Kliniczno-Badawcze J. Brzezicki, B., Gornikiewicz Brzezicka Lekarze Spolka Partnerska, ul. Studzienna 35-36A, Elblag). Spain: Merce Falip (Hospital de Bellvitge, Neurology Department, Barcelona); Rodrigo Rocamora (Hospital del Mar, Neurology Department, Barcelona). United Kingdom: Manny Bagary (Regional Epilepsy Clinic, BSMHFT, Birmingham); Carlo Canepa (James Paget University Hospitals NHS Foundation Trust, Great Yarmouth, Norfolk).

\section{Author Disclosure Statement}

M.N., F.S., and A.S. are employed by GW Research Ltd and hold share options in GW Research Ltd. L.P. has received consultation fees from GL Pharma, Mylan, Sandoz, and Vipharm. He has also received educational grants and support from Vipharm, GP Pharma, and Mylan. He has accepted honoraria and guest lecture fees for symposia and other events sponsored by Astellas, Lundbeck, Teva, Eisai, and GL Pharma. He has served as principal investigator for $>50$ sponsorinitiated studies, and has been a national coordinator and key opinion leader for Astellas. He has served as an expert witness and consultant for lawsuits, providing deposition and testimony, including analysis of medical documents. J.W.S. received research grants from UCB and personal fees from UCB and Zogenix outside the submitted work. P.C. has served mainly as principal investigator in 66 industry-sponsored clinical trials in epilepsy and migraine. In seven trials, he was country coordinator. He has co-organized 26 annual conferences, named Psychosocial Aspects of Epilepsy sponsored by Pfizer, Glaxo, and Sanofi in the past and UCB Pharma, Teva, Apotex, Mylan, Glenmark, Accord, and Novartis currently. He has accepted speaking fees for lectures and webinars sponsored by UCB Pharma.

\section{Funding Information}

The trial was sponsored by GW Research Ltd.

\section{Supplementary Material}

Supplementary Data

\section{References}

1. Hillig KW, Mahlberg PG. A chemotaxonomic analysis of cannabinoid variation in Cannabis (Cannabaceae). Am J Bot. 2004;91:966975 . 
2. Hill TD, Cascio MG, Romano B, et al. Cannabidivarin-rich cannabis extracts are anticonvulsant in mouse and rat via a CB1 receptor-independent mechanism. Br J Pharmacol. 2013;170:679-692.

3. lannotti FA, Hill CL, Leo A, et al. Nonpsychotropic plant cannabinoids, cannabidivarin (CBDV) and cannabidiol (CBD), activate and desensitize transient receptor potential vanilloid 1 (TRPV1) channels in vitro: potential for the treatment of neuronal hyperexcitability. ACS Chem Neurosci. 2014;5:1131-1141.

4. Huizenga MN, Sepulveda-Rodriguez A, Forcelli PA. Preclinical safety and efficacy of cannabidivarin for early life seizures. Neuropharmacology. 2019;148:189-198.

5. Posner K, Brent D, Lucas C, et al. Columbia-Suicide Severity Rating Scale (C-SSRS). New York State Psychiatric Institute: New York, NY, 2008.

6. Allsop DJ, Norberg MM, Copeland J, et al. The Cannabis Withdrawal Scale development: patterns and predictors of cannabis withdrawal and distress. Drug Alcohol Depend. 2011;119:123-129.

7. Guekht $A B$, Korczyn AD, Bondareva IB, et al. Placebo responses in randomized trials of antiepileptic drugs. Epilepsy Behav. 2010;17:64-69.

8. Burneo JG, Montori VM, Faught E. Magnitude of the placebo effect in randomized trials of antiepileptic agents. Epilepsy Behav. 2002;3:532534.

9. Cramer JA, Fisher R, Ben-Menachem E, et al. New antiepileptic drugs: comparison of key clinical trials. Epilepsia. 1999;40:590600.

10. Zaccara G, Giovannelli F, Cincotta M, et al. Adverse events of placebotreated, drug-resistant, focal epileptic patients in randomized controlled trials: a systematic review. J Neurol. 2015;262:501-515.

11. Goldenholz DM, Goldenholz SR. Response to placebo in clinical epilepsy trials-Old ideas and new insights. Epilepsy Res. 2016;122:15-25.
12. French JA, Cabrera J, Emir B, et al. Designing a new proof-of-principle trial for treatment of partial seizures to demonstrate efficacy with minimal sample size and duration-a case study. Epilepsy Res. 2013;106:230-223.

Cite this article as: Brodie MJ, Czapinski P, Pazdera L, Sander JW, Toledo M, Napoles M, Sahebkar F, Schreiber A; on Behalf of the GWEP1330 Study Group (2021) A phase 2 randomized controlled trial of the efficacy and safety of cannabidivarin as add-on therapy in participants with inadequately controlled focal seizures, Cannabis and Cannabinoid Research 6:6, 528-536, DOI: 10.1089/can.2020.0075. 